\title{
Monte Carlo Simulations for Quantum Field Theories Involving Fermions
}

\author{
M. Karowski ${ }^{1 \star}$, R. Schrader ${ }^{2 \star}$, and H. J. Thun ${ }^{3}$ \\ 1 Institut für Theoretische Physik, Universität Göttingen, Bunsenstrasse 9, D-3400 Göttingen, \\ Federal Republic of Germany \\ 2 State University of New York at Stony Brook, Stony Brook, NY 11794, USA \\ 3 Institut für Theorie der Elementarteilchen, Freie Universität Berlin, Arnimallee 14, \\ D-1000 Berlin 33
}

\begin{abstract}
We present a new variant of a Monte Carlo procedure for euclidean quantum field theories with fermions. On a lattice every term contributing to the expansion of the fermion determinant is interpreted as a configuration of self-avoiding oriented closed loops which represent the fermionic vacuum fluctuations. These loops are related to Symanzik's polymer description of euclidean quantum field theory. The method is extended to the determination of fermionic Green's functions. We test our method on the Scalapino-Sugar model in one, two, three, and four dimensions. Good agreement with exactly known results is found.
\end{abstract}

\section{Introduction}

In recent years Monte Carlo simulations for euclidean lattice models have been of considerable help in improving our understanding of those relativistic quantum field theories, which are supposed to describe high energy particle physics. This includes in particular gauge theories. Now any realistic model for particle interactions includes fermionic fields like quark fields. It is, therefore, important to simulate systems with fermionic degrees of freedom. There have been several proposals to deal with this problem, see e.g. [1-16], or [17-19] for a review. However, all these methods require extensive computing time and some of them only work for two-dimensional models or are only approximations from the beginning: quenched approximation, hopping parameter expansion etc. In particular for the interesting case of four-dimensional lattices no way has yet been found to perform Monte Carlo calculations including fermions as efficiently as they can be done when bosonic fields only are present.

It is the aim of this paper to propose a new numerical method which basically treats all fields on the same footing during the upgrading procedure. This new way of treating fermions applies to all lattice models known to the authors and may

* On leave of absence from Freie Universität Berlin 
easily be combined with the standard methods already used for bosonic fields. We now briefly describe our method, the details of which will be given in the next sections. To deal with the fermionic degrees of freedom we introduce an auxiliary statistical ensemble, the elements of which are labelled by a subset of the set of permutations on the fermionic degrees of freedom. We give a graphical representation of such an element of the statistical ensemble in terms of selfavoiding loops and relate it to Symanzik's polymer description of euclidean quantum field theory [20,21]. This procedure guarantees that Pauli's exclusion principle for the fermions is automatically taken care of. The antisymmetry in the fermionic degrees of freedom is obtained by equating the Green's functions of the physical model with certain (ratios of) Green's functions of the auxiliary statistical ensemble. This is explained in Sect. 2.

In Sect. 3 we present a local heat bath method for a fermionic action in the form originally introduced by Kogut and Susskind in a Hamiltonian context [22]. As just mentioned, each element of the statistical ensemble, which is a certain permutation, has a graphical representation. The heat bath is local, i.e. most of the fermionic degrees of freedom are frozen, because we apply localized permutations which upgrade a given permutation only, locally. The intricate part of our procedure is to show that the principle of detailed balance is satisfied. This combined with the ergodicity of the upgrading ensures that the Gibbs distribution for the auxiliary statistical ensemble is the unique equilibrium distribution for the upgrading procedure.

In Sect. 4 we consider the standard, simple model introduced by Scalapino and Sugar [3], which has often been used to test methods for dealing with fermions numerically. We compare our Monte Carlo results with exact values obtained by numerical Fourier summation. Finally, Sect. 5 contains some conclusions.

\section{Statistical Ensembles for Fermions}

In this section we will discuss the auxiliary statistical ensemble, in terms of which the fermionic degrees of freedom may be described so as to give the Green's functions of the physical theory. We begin by recalling the standard formulation of lattice theories involving fermions. We will restrict our attention to (finite) cubical lattices in $d$ dimensions. The question of how to choose the right boundary conditions will not be relevant in this section.

Consider a lattice action of the form

$$
S=S\left(\psi^{+}, \psi, \phi\right)
$$

$\psi^{+}$and $\psi$ denote fermionic fields and are considered to be Grassmann variables. $\phi$ stands for all other fields, which are supposed to be bosonic. In all applications so far, the fermionic fields are defined on the vertices of the lattice. Thus the lattice site $x$ may be used to label the fermionic degrees of freedom. The fields $\phi$ may live on vertices (ordinary Bose fields) or on links (lattice gauge fields). Our method will also allow for more complicated situations, where for example $\phi$ lives on higher dimensional cells. 
For the purpose of explaining our method, we first look at the simplified situation, where

(a) the fermionic fields carry no indices (like flavour or colour) other than the vertex index,

(b) the action is quadratic in the fermionic fields.

It is easy to extend our method to situations, where these restrictions are removed and at the end of the section we will briefly indicate how this is done. With these restrictions the action $S$ may be decomposed as

$$
S\left(\psi^{+}, \psi, \phi\right)=S_{F}\left(\psi^{+}, \psi, \phi\right)+S_{B}(\phi),
$$

where

$$
S_{F}\left(\psi^{+}, \psi, \phi\right)=\sum_{x, y \in \text { Lattice }} \psi^{+}(x) A_{x y}(\phi) \psi(y) .
$$

Here the matrix $A=A(\phi)=\left\{A_{x y}(\phi)\right\}$ with complex valued entries is indexed by the vertices of the lattice and is a functional of the bosonic fields $\phi$, and $S_{B}(\phi)$ is that part of the action which does not involve fermionic fields. The "partition" function of the theory is therefore

$$
Z_{F B}=\int d \phi d \psi^{+} d \psi e^{-S} .
$$

The integration over the fermionic fields is in the sense of Berezin [23], and $d \phi$ describes the integration over the bosonic fields $\phi$. By

$$
\langle X\rangle=Z_{F B}^{-1} \int d \phi d \psi^{+} d \psi X e^{-S}
$$

we denote the expectation value in this model. If we perform the fermionic integration first, Eq. (2.4) may be written as

$$
Z_{F B}=\int d \phi \operatorname{det} A(\phi) e^{-S_{B}(\phi)} .
$$

To obtain Green's functions involving fermionic fields, we consider the typical example $\left\langle\psi(v) \psi^{+}(u)\right\rangle$. For given $u$, $v$ let $A^{(u, v)}$ denote the matrix given by

$$
A_{x y}^{(u, v)}= \begin{cases}A_{x y} & \text { if } x \neq u \text { and } y \neq v \\ 1 & \text { if } x=u \text { and } y=v \\ 0 & \text { otherwise }\end{cases}
$$

Then we have

$$
\left\langle\psi(v) \psi^{+}(u)\right\rangle=\frac{\int d \phi \operatorname{det} A^{(u, v)}(\phi) e^{-S_{B}(\phi)}}{\int d \phi \operatorname{det} A(\phi) e^{-S_{B}(\phi)}} .
$$

Of course, $\operatorname{det} A^{(u, v)}$ is (up to a sign) equal to the determinant of the submatrix of $A$ obtained by deleting the $u^{\text {th }}$ row and $v^{\text {th }}$ column. More generally, any (higher order) Green's function in the fermionic fields may be obtained in this way from determinants of suitable submatrices. Formulas (2.6) and (2.8) are obtained from Berezin's integration theory, by using the standard formula

$$
\operatorname{det} A=\sum_{\pi} \operatorname{sgn}(\pi) \prod_{x} A_{x \pi(x)}
$$

where $\pi$ runs through the set of all permutations of lattice points. 
Let us now neglect the $\phi$ dependence for a moment. Each term on the righthand side of (2.9), which is labelled by $\pi$, may be given a graphical presentation as follows. If $\pi(x) \neq x$, we draw an oriented straight line in the lattice from the point $x$ to the point $\pi(x)$. By definition its length is 1 . Now every permutation may be written as a product of (nontrivial) cyclic permutations

$$
\pi=\pi_{1} \ldots \pi_{s}
$$

and this description is unique up to the ordering of the cyclic factors $\pi_{r}, 1 \leqq r \leqq s$. If $\ell_{r}$ denotes the order of $\pi_{r}$ (such that $\ell_{r}=2$ if $\pi_{r}$ is a transposition), we have

$$
\operatorname{sgn}(\pi)=\prod_{r=1}^{s}(-1)^{\ell_{r}+1}
$$

Using this graphical presentation each cyclic permutation $\pi_{r}$ corresponds to a closed oriented polygonal loop $\mathscr{L}_{r}$ of length $\ell_{r}$. These loops $\mathscr{L}_{1}, \ldots, \mathscr{L}_{s}$ are nonintersecting in the sense that each vertex in the lattice is the endpoint of at most one oriented straight line and in that case it is also the starting point of exactly one line.

Conversely to each such family of nonintersecting oriented polygonal loops corresponds a unique $\pi$ and hence a unique contribution to det $A$ in the sense of Eq. (2.9). Note that we only need to consider those $\pi$ which are contained in the set

$$
\mathscr{C}(A)=\left\{\pi ; \prod_{x} A_{x \pi(x)} \neq 0\right\} .
$$

Similarly, the only nonvanishing contributions to $\operatorname{det} A^{(u, v)}$ are among those $\pi$ for which $\pi(u)=v$. By deleting the particular straight line going from $u$ to $v$, each such contribution to $\operatorname{det} A^{(u, v)}$ can be graphically described by a set of nonintersecting loops plus an additional "propagator", i.e. an open polygonal line, going from $v$ to $u$, which is nonselfintersecting and not intersecting the other loops in the sense just described.

It is important to note that this nonintersecting property is a local property, i.e. it is only necessary to test all vertices individually to see whether a given set of polygonal loops correspond to a permutation $\pi$ or not.

In most applications the matrix $A$ will have the following additional property: $A$ is said to be local, if $A_{x y}=0$ unless $\operatorname{dist}(x, y) \leqq 1$. In the context of our graphical presentation this means that all loops are built out of links. Also the nonintersecting property stated above is now the usual nonintersecting property of curves. For local $A$, we say $\pi \in \mathscr{C}(A)$ is a "dimer" if $\pi$ is cyclic of order 2. $\pi$ is said to contain a dimer if at least one of its cyclic factors $\pi_{r}$ is of order two. Now for local $A$, the number $|\mathscr{C}(A)|$ of elements in $\mathscr{C}(A)$ is bounded above by $(2 d+1)^{\text {(volume of lattice) }}$. On the other hand, since the density of states for the dimer problem is explicitly known for $d=2$ at zero temperature [24-27], it is easy to obtain the lower bound $\alpha^{\text {volume } / 2}$ on $|\mathscr{C}(A)|\left(\alpha=\exp \frac{2 G}{\pi}=1.791 \ldots, G=\right.$ Catalan's $=0.915 \ldots)$, if $A$ is such that $A_{x y} \neq 0$ whenever $\operatorname{dist}(x, y) \leqq 1$. Indeed, this follows by 
considering the subset of $\mathscr{C}(A)$ consisting of those $\pi$ with dimers only which point in directions parallel to a given plane. In Sect. 3 we will also give a discussion on how to compute $|\mathscr{C}(A)|$ numerically for the case of such a local $A$.

The graphical presentation we have given for general (constant) matrix $A$ is related to Symanzik's polymer presentation [20, 21], see also [28, 29], as follows. For simplicity, let $A$ be of the special form

$$
A=\mathbb{1}+\Gamma,
$$

with $\Gamma_{x x}=0$ and $\Gamma_{x y}$ small. Then

$$
\begin{aligned}
\operatorname{det} A & =\exp [\operatorname{tr} \ln A] \\
& =\exp \left[\sum_{n=1}^{\infty} \frac{(-1)^{n-1}}{n} \operatorname{tr} \Gamma^{n}\right] .
\end{aligned}
$$

Since

$$
\operatorname{tr} \Gamma^{n}=\sum_{x_{1}, \ldots, x_{n}} \Gamma_{x_{1} x_{2}} \ldots \Gamma_{x_{n} x_{1}} .
$$

each term of the right-hand side of (2.15) can be viewed graphically as an oriented polygonal loop of length $n$. In this formulation, however, there is no nonintersecting condition, i.e. each vertex may be the endpoint of more than one oriented straight line. The converse holds also in this case: To each such loop of length $n$ corresponds a unique contribution to $\operatorname{tr} \Gamma^{n}$ via (2.15). The Taylor expansion of the exponent in Eq. (2.14) then gives a combinatorial description (and proof) of how these terms in (2.14)-(2.15), each of which is presented by one oriented loop, combine to the set of terms in (2.9), each of which is presented by a family of nonintersecting oriented loops. A similar graphically equivalent description may be given for the Green's functions, e.g. $\left\langle\psi(v) \psi^{+}(u)\right\rangle$.

We now turn to a construction of the statistical ensemble. Let the matrix $|A|$ be given by

$$
|A|_{x y}=\left|A_{x y}\right|
$$

and consider the permanent of $|A|$,

$$
\operatorname{Per}(|A|)=\sum_{\pi \in \mathscr{C}(A)} \prod_{x}|A|_{x \pi(x)} .
$$

Now $\mathscr{C}(A)$ may be viewed as a Gibbs statistical ensemble with energies given by

$$
E(\pi, A)=\sum_{x} E_{x}(\pi, A)=-\sum_{x} \ln \left(|A|_{x \pi(x)}\right),
$$

such that the permanent becomes the corresponding partition function

$$
\operatorname{Per}(|A|)=\sum_{\pi \in \mathscr{C}(A)} \exp [-E(\pi, A)]
$$

If we denote by

$$
\bar{X}=\frac{1}{\operatorname{Per}(|A|)} \cdot \sum_{\pi} X(\pi) \cdot e^{-E(\pi, A)}
$$


averages in this statistical ensemble, we have the following relation for the determinant of $A$

$$
\begin{aligned}
\operatorname{det} A & =\sum_{\pi} \operatorname{sgn}(\pi, A) e^{-E(\pi, A)} \\
& =\operatorname{Per}(|A|) \cdot \overline{\operatorname{sgn}(A)}
\end{aligned}
$$

where $\operatorname{sgn}(\pi, A)$ is defined on $\mathscr{C}(A)$ by

$$
\operatorname{sgn}(\pi, A)=\operatorname{sgn}(\pi) \cdot \prod_{x} \frac{A_{x \pi(x)}}{\left|A_{x \pi(x)}\right|}
$$

To obtain the Green's function $\left\langle\psi(v) \psi^{+}(u)\right\rangle$ we have to determine [cf. Eq. (2.8)]

$$
\operatorname{det} A^{(u, v)}=\operatorname{Per}\left(\left|A^{(u, v)}\right|\right) \cdot \operatorname{sgn}\left(A^{(u, v)}\right),
$$

where now the configurations of $\mathscr{C}\left(A^{(u, v)}\right)$ have to be taken into account.

We remark that the following modification of this construction leads to the theory of the noninteracting polymer gas: Take $A$ to be local with $A_{x x}=1$ and $A_{x y}=$ const for $\operatorname{dist}(x, y)=1$. The statistical ensemble is defined to be the subset of $\mathscr{C}(A)$ consisting of all $\pi$ 's without dimers. In fact, the numerical results in [42] were obtained by a corresponding modification of the upgrading procedure, to be explained in Sect. 3.

Let us return to the case, where bosonic fields $\phi$ are present. Again we define $\mathscr{C}(A)$ by (2.12), where the condition $\prod_{x} A_{x \pi(x)} \neq 0$ is now understood in the sense of functionals. Also in (2.18) we set $E_{x}(\pi, A(\phi))=\infty$ for any value of $\phi$ for which $A_{x \pi(x)}(\phi)=0$. We are now in a position to describe the auxiliary statistical ensemble and express physical Green's functions as expectation values in this theory. In fact, the thermal average values are now

$$
\bar{X}=Z^{-1} \int d \phi \sum_{\pi \in \mathscr{C}(A)} X(\pi, \phi) e^{-S_{B}(\phi)-E(\pi, A(\phi))},
$$

with

$$
Z=\int d \phi \sum_{\pi \in \mathscr{C}(A)} e^{-S_{B}(\phi)-E(\pi, A(\phi))}
$$

The set of configurations is now the product $\mathscr{C}(A)$ times the set of the usual bosonic configurations. Consider first an observable $F$ which only depends on $\phi$. Then

$$
\begin{aligned}
\langle F(\phi)\rangle & =\frac{\int d \phi F(\phi) \operatorname{det} A(\phi) e^{-S_{B}(\phi)}}{\int d \phi \operatorname{det} A(\phi) e^{-S_{B}(\phi)}} \\
& =\frac{\int d \phi \sum_{\pi \in \mathscr{C}(A)} F(\phi) \operatorname{sgn}(\pi, A(\phi)) e^{-S_{B}(\phi)-E(\pi, A(\phi))}}{\int d \phi \sum_{\pi \in \mathscr{C}(A)} \operatorname{sgn}(\pi, A(\phi)) e^{-S_{B}(\phi)-E(\pi, A(\phi))}} \\
& =\frac{\bar{F} \cdot \operatorname{sgn}(A) / \operatorname{sgn}(A)}{}
\end{aligned}
$$


We note a crucial property of this relation, which will allow us to perform local upgrading procedures both for the fermionic and the bosonic part: $E(\pi, A(\phi))$ is a sum of local terms $E_{x}(\pi, A(\phi))$ in the sense that for local theories the latter will depend only on the form of $\pi$ near $x$ and on the field configurations of $\phi$ which live near $x$.

Next we turn to fermionic Green's functions. For the two-point function we obtain as a generalization of Eq. (2.23) [see Eq. (2.8)]

$$
\left\langle\psi(v) \psi^{+}(u)\right\rangle=\frac{\overline{\operatorname{sgn}\left(A^{(u, v)}\right)}}{\overline{\operatorname{sgn}(A)}} .
$$

Analogously higher order Green's functions may be obtained by augmenting the statistical ensemble appropriately.

The remainder of this section is devoted to a brief outline of the modifications necessary to cover the cases that

(a) the fermionic fields carry indices $\varrho$ which may include the vertex index $x$ and internal degrees of freedom and

(b) higher order interactions in the fermionic fields are present.

In the case (a), which is important for treating e.g. non-abelian lattice gauge theories, $A$ is a matrix $A_{\rho x}$. And $\mathscr{C}(A)$ is now defined to be the set of all permutations $\pi$ of the $\varrho$ 's for which $\prod_{\pi} A_{\varrho \pi(\varrho)}(\phi) \neq 0$. Again there is a graphical representation which now is in $d^{\prime}$ dimensions $\left(d^{\prime}>d\right)$ with the extra $\left(d^{\prime}-d\right)$ dimensions being used to describe the additional degrees of freedom.

As for the case (b) assume the action contains an extra term which is of fourth order in the fermionic fields

$$
S_{F}^{\prime}\left(\psi^{+}, \psi, \phi\right)=\sum_{\substack{0^{\neq} \varrho^{\prime} \\ x \neq \chi^{\prime}}} \psi^{+}(\varrho) \psi^{+}\left(\varrho^{\prime}\right) B_{\varrho \varrho^{\prime} x x^{\prime}} \psi(\varkappa) \psi(\varkappa) .
$$

Let $\mathscr{J}=\left(I, I_{1}, \ldots, I_{k}\right)(0 \leqq k)$ be a decomposition of the set of all $\varrho$ 's into a subset $I$ and ordered sets $I_{l}(1 \leqq \ell \leqq k)$ containing two elements each. Then

$$
\begin{aligned}
& \int d \psi^{+} d \psi e^{-S_{F}\left(\psi^{+}, \psi, \phi\right)-S_{F}^{\prime}\left(\psi^{+}, \psi, \phi\right)} \\
& =\sum_{\pi, \mathscr{g}} \operatorname{sgn}(\pi) \prod_{\varrho \in I} A_{\varrho \pi(\varrho)}(\phi) \prod_{\substack{\ell=1 \\
\left(\varrho^{\prime}, \varrho^{\prime \prime}\right)=I_{\ell}}}^{k} B_{\varrho^{\prime} \varrho^{\prime \prime} \pi\left(\varrho^{\prime}\right) \pi\left(\varrho^{\prime \prime}\right)}(\phi) .
\end{aligned}
$$

The auxiliary statistical ensemble is now labelled by $\pi, \mathscr{J} \in \mathscr{C}(A, B)$, and the states representing the bosonic fields $\phi$, where

$$
\mathscr{C}(A, B)=\left\{(\pi, \mathscr{J}) ; \prod_{\varrho \in I} A_{\varrho \pi(\varrho)} \prod_{\substack{\ell=1 \\\left(\varrho, \varrho^{\prime}\right)=I_{\ell}}}^{k} B_{\varrho \varrho^{\prime} \pi(\varrho) \pi\left(\varrho^{\prime}\right)} \neq 0\right\} .
$$

Also the energies (2.18) are replaced by

$$
E(\pi, \mathscr{J}, A(\phi), B(\phi))=-\sum_{\varrho \in I} \ln \left|A_{\varrho \pi(\varrho)}(\phi)\right|-\sum_{\substack{\ell=1 \\\left(\varrho, \varrho^{\prime}\right)=I_{\ell}}}^{k} \ln \left|B_{\varrho \varrho^{\prime} \pi(\varrho) \pi\left(\varrho^{\prime}\right)}(\phi)\right| .
$$

Again a graphical presentation may be obtained. It is obvious how to extend this procedure to interactions of order higher than four. 
Another strategy would work for local and translation invariant $B$ 's. One can introduce an intermediate boson $\varphi(\varrho, \chi)$ and replace $(2.28)$ by

$$
\begin{aligned}
& S_{F}^{\prime \prime}\left(\psi^{+}, \psi, \varphi, \phi\right) \\
& \quad=-\sum\left[\varphi(\varrho, x) B_{\varrho \varrho^{\prime} x x^{\prime}} \varphi\left(\varrho^{\prime}, x^{\prime}\right)+\psi^{+}(\varrho) \psi(x)\left(B_{\varrho \varrho^{\prime} x x^{\prime}}+B_{\varrho^{\prime} \varrho x / x}\right) \varphi\left(\varrho^{\prime}, x^{\prime}\right)\right],
\end{aligned}
$$

which again turns out to be an action of case $\left(\mathrm{a}^{\prime}\right)$.

\section{The Heat Bath Method}

In this section we explain our heat bath method for the determination of fermion determinants for the case of free massive fermions.

There exist different formulations for fermions on a lattice, e.g. the Wilson [30], Kogut-Susskind [31-36], and Dirac-Kähler [37-39] versions. For the purpose of this paper the second version is the most adequate one. Thus we use it to exemplify our heat bath method in spite of its shortcoming, namely numerical results are reliable only for sufficiently heavy fermions. More precisely, the statistical errors obscure the measurements if the hopping parameter $k=1 /(2 \mathrm{am})$ ( $a=$ lattice spacing, $m=$ fermion mass) exceeds $0.6,0.25,0.15$ for Susskind fermions in 2, 3, and 4 dimensions, respectively. In a forthcoming publication we shall extend our method to the case of Wilson fermions, where there is no such restriction on the mass.

We consider a hypercubic lattice in $d$ dimensions with lattice points

$$
\mathbf{x}=\sum_{\mu=1}^{d} x_{\mu} \mathbf{a}_{\mu}
$$

labelled by integer components, $x_{\mu}=0,1,2, \ldots, L-1$, and $\left\{\mathbf{a}_{\mu}\right\}$ orthogonal vectors parallel to the lattice axes, $\mathbf{a}_{\mu} \cdot \mathbf{a}_{v}=a^{2} \delta_{\mu v}$. The naive lattice version of the euclidean free fermion action reads

with central differences, i.e.

$$
S=\sum_{x} \psi^{+}(x)\left(\gamma_{\mu} \partial_{\mu}+m\right) \psi(x)
$$

$$
\partial_{\mu} \psi(x)=\frac{1}{2 a}\left[\psi\left(x+a_{\mu}\right)-\psi\left(x-a_{\mu}\right)\right]
$$

The Susskind formulation is most easily obtained by the transformation $[35,36]$

$$
\psi(x) \rightarrow \prod_{\mu=1}^{d} \gamma_{\mu}^{x_{\mu}} \psi(x)
$$

the $\gamma$-matrices then become proportional to the unit matrix, albeit $x$-dependent,

$$
\gamma_{\mu} \rightarrow \gamma_{\mu}(x) \mathbb{1}=\prod_{v=1}^{\mu-1}(-1)^{x_{v}} \mathbb{1}
$$

It is then sufficient to consider only one component of a full Dirac fermion at each lattice site, and the matrix which appears in Eq. (2.3) takes the form

$$
\left(\gamma_{\mu} \partial_{\mu}+m\right)_{\mathbf{x}, \mathbf{y}}=\gamma_{\mu}(x) \frac{1}{2 a}\left[\delta_{\mathbf{x}+\mathbf{a}_{\mu}, \mathbf{y}}-\delta_{\mathbf{x}-\mathbf{a}_{\mu}, \mathbf{y}}\right]+m \delta_{\mathbf{x}, \mathbf{y}} .
$$


The fermion determinant is therefore given by

$$
\operatorname{det}(\gamma \partial+m)=\sum_{\pi \in S_{N}} \operatorname{sgn}(\pi) \prod_{x}(\gamma \partial+m)_{x \pi(x)},
$$

with $N=L^{d}=$ number of lattice points.

As explained in Sect. 2 there is a one-to-one correspondence between the permutation $\pi \in S_{N}$ of the lattice points and loop configurations $c \in \mathscr{C}(\gamma \partial+m)$ on the lattice, therefore,

$$
Z_{F} \equiv \operatorname{det}(\gamma \partial+m)=m^{N} \sum_{c \in \mathscr{C}} \operatorname{sgn}(c, \gamma \partial+m) k^{\ell(c)}
$$

[with $k=1 /(2 m)$, and we set $a=\left|\mathbf{a}_{\mu}\right|=1$ from now on]. The sum runs over all configurations $c$ of self-avoiding closed oriented loops on the lattice made up from single links ("monomers") connecting neighbouring sites.

The integer $\ell(c)$ is the total length in lattice units of all loops in the configuration $c$. The smallest allowed loops are "dimers" with $\ell=2$, righthanded and lefthanded $1 \times 1$ square loops with $\ell=4$, etc. There is a direct geometrical interpretation of $\operatorname{sgn}(c, \gamma \partial+m)$, which has been defined in Eq. (2.21). For a single loop $\operatorname{sgn}(c, \gamma \partial+m)=(-1)^{n_{i}}$, where $n_{i}$ is the number of internal lattice points in a surface (made up of plaquettes) whose boundary is the loop $c$. This implies that in $d=1$ dimension there are no negative contributions to Eq. (3.6) at all, whereas in $d=2$ dimensions the simplest ones are due to $2 \times 2$ square loops with $n_{1}=1, \ell=8$, and in $d \geqq 3$ they arise from certain nonplanar loops with $\ell=6$. Such negative contributions give rise to large statistical errors in the evaluation of expectation values like (2.20) and (2.22) by Monte Carlo methods. This fact is responsible for the empirically determined "critical values" for $k$ mentioned at the beginning of this section.

Next we introduce the partition function of an auxiliary statistical mechanics problem of a gas of self-avoiding closed orientated loops,

$$
Z(k)=\sum_{c \in \mathscr{C}} k^{\ell(c)},
$$

where $k=e^{-\beta}$ now is the "monomer" fugacity ( $\beta=$ inverse temperature). In terms of the thermal average of an observable $X$ for this auxiliary problem,

$$
\bar{X}=Z^{-1} \sum_{c \in \mathscr{C}} X(c) k^{\ell(c)}
$$

we then can rewrite the fermion determinant Eq. (3.6) as

$$
Z_{F}=m^{N} \cdot Z \cdot \overline{\operatorname{sgn}(\gamma \partial+m)},
$$

and the quantum field vacuum expectation value is

$$
\langle X\rangle=Z_{F}^{-1} \int d \psi^{+} d \psi X \cdot e^{-S}=\overline{X \cdot \operatorname{sgn}} / \overline{\operatorname{sgn}} .
$$

The partition function $Z$ itself can be obtained from the average energy $\bar{\ell}$. In fact, Eq. (3.7) gives

$$
k \frac{\partial}{\partial k} \ln Z(k)=\bar{\ell}(k)
$$


and by integration

$$
Z(k)=\exp \int_{0}^{k} \frac{d k^{\prime}}{k^{\prime}} \bar{\ell}\left(k^{\prime}\right)
$$

In particular, we can evaluate Eq. (3.12) for $k=1$ to get the number of all configurations in $\mathscr{C}$ [cf. Eq. (3.7)].

Similarly, the fermion determinant is computable from $\langle\ell\rangle$,

$$
Z_{F}(k)=m^{N} \exp \int_{0}^{k} \frac{d k^{\prime}}{k^{\prime}}\langle\ell\rangle\left(k^{\prime}\right),
$$

which is obtained by differentiating Eq. (3.6) with respect to $k=1 / 2 m$,

$$
k \frac{\partial}{\partial k} \ln Z_{F}(k)=\overline{\ell \cdot \operatorname{sgn}} / \overline{\operatorname{sgn}}-N=\langle\ell\rangle-N .
$$

It is now clear that it is sufficient to know the average values $\bar{\ell}, \overline{\operatorname{sgn}}, \overline{\ell \cdot \operatorname{sgn}}$, etc. for the auxiliary problem. They can be determined numerically by means of a Monte Carlo simulation $[40,41]$. In the remainder of this section we shall explain in some detail our variant of the heat bath method which generates samples of equilibrium ensembles of configurations in $\mathscr{C}(\gamma \partial+m)$.

Starting from an arbitrary allowed old configuration $c_{0}$ of loops with total length $\ell_{0}$ a new configuration $c_{n}$ with length $\ell_{n}$ is proposed. We accept the new one with probability

$$
w=\frac{w_{n}}{w_{0}+w_{n}},
$$

where

$$
w_{0, n}=k^{\ell_{0, n}}
$$

are the Boltzmann weights of the old and new configurations, respectively. In fact, we actually take the new configuration $c_{n}$ if a pseudo-random number (equally distributed in the unit interval) is less than $w$, otherwise, we retain the old configuration $c_{0}$. In our updating procedure for the creation of new configurations from old ones we systematically sweep the plaquettes of the entire lattice. At each of the $N=L^{d}$ lattice points we look in turn at $d(d-1) / 2$ plaquettes. In a given plaquette we then perform tentative local changes of the old configuration.

In practice we do not use the components (3.1) to specify a lattice point, but number them lexicographically by the integers

$$
x=\sum_{\mu=1}^{d} L^{\mu-1} x_{\mu}
$$

i.e. $x=0,1, \ldots, N-1$.

As explained above each configuration of loops $c \in \mathscr{C}$ corresponds to a permutation $\pi \in S_{N}$ and thus gives rise to a term in the expansion of the fermion 
determinant, Eq. (3.5). It is instructive to think of the permutations in the following way. Imagine e.g. a "dog" sitting at each (lattice) site. In the identical permutation every dog is at home, $\pi(x)=x$. Because of chains of unit length the dogs cannot go beyond their next neighbor's sites. The neighbor then has to look for another place, too. By a permutation $\pi$ we mean that a dog moves from $x$ to $\pi(x)$, therefore, $\pi^{-1}(x)$ is the site wherefrom the dog, now sitting at $x$, has come. It is convenient to parametrize the configuration by

$$
p(x)=\pi^{-1}(x)-x
$$

which is the distance (in site numbers) of the home site of a dog from its actual position. Because of the nearest neighbor property of the matrix $\gamma \partial+m$, i.e. the chains, the function $p(x)$ is restricted to $(2 d+1)$ values

$$
p(x)=0, \pm 1, \pm L, \ldots, \pm L^{d-1}
$$

for allowed configurations. In this picture the links of the self-avoiding closed loops are represented by the chains of those dogs which are not at home.

Let us now explain in more detail how we generate a new configuration from an allowed old one by a rearrangement within a given plaquette. We work with a set of 14 local operations described in terms of permutations and defined on a plaquette, $q_{1}, q_{2}, \ldots, q_{14}$. They fall into four classes of cyclic permutations. Elements in one class are obtained from one another by rotations and reflections. Figure 1 gives a representative of each class.

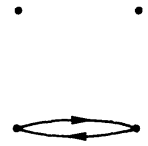

a

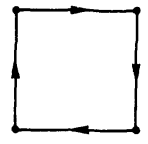

b

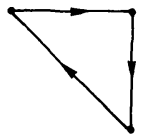

C

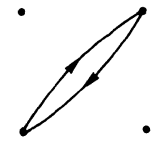

d

Fig. 1a-d. Representatives of the four classes of local permutations

So these four classes contain $2,2,8$, and 2 operations, respectively.

A tentative new permutation $\pi_{n}$ is obtained from an old one $\pi_{0}$ by

$$
\pi_{0} \rightarrow \pi_{n}=q_{j} \circ \pi_{0} ; \quad j=1,2, \ldots, 14
$$

which implies

$$
p_{n}(x)=p_{0}\left(q_{j}^{-1}(x)\right)+q_{j}^{-1}(x)-x
$$

The new permutation is allowed, i.e. compatible with the nearest neighbor property, if and only if $p_{n}(x)$ takes permissible values, cf. (3.19). This has to be checked only for the points in the plaquette since they are the only ones affected by the rearrangement. For $p_{n}(x)$ unequal to the specific values (3.19) the operation would tear a dog too far from its home site and the corresponding matrix element in that contribution to the right-hand side of Eq. (3.5) would vanish. 
We take into consideration the different possibilities $j=1,2, \ldots, 14$ by the following strategy. First, we deal with the creation or annihilation of "dimers" corresponding to Fig. 2 a

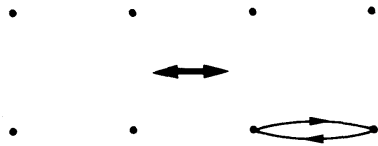

a

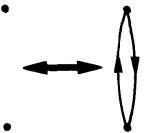

b

Fig. 2a and b. Dimer creation and annihilation

which result from the application of the two-cycle operation $q_{1}$ of Fig. 1a. If the new configuration is allowed we accept it with probability given by Eqs. (3.15) and (3.16). We repeat this procedure with the operation $q_{2}$ (a rotated version of $q_{1}$ ).

If after these manipulations the plaquette indeed contains a dimer, we immediately turn to the next plaquette. Otherwise, we try to apply one of the remaining operations $q_{3}, \ldots, q_{14}$ corresponding to Fig. $1 \mathrm{~b}-\mathrm{d}$. The resulting four types of transitions are depicted in Fig. 3.

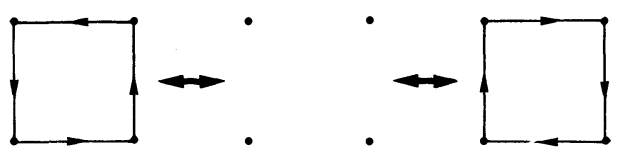

a

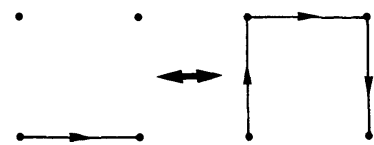

b

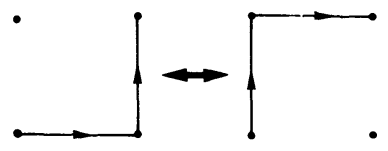

C

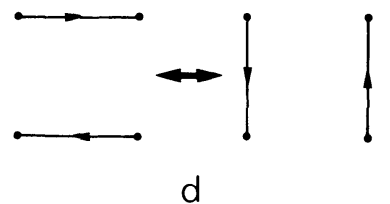

Fig. 3a-d. Local changes of loop configurations not involving dimers 
Type (a) is generated by four-cycle operations $q_{3}$ and $q_{4}$ corresponding to Fig. $1 \mathrm{~b}$, types (b) and (c) are due to three-cycles $q_{5}, \ldots, q_{12}$, cf. Fig. 1c, and finally, type (d) originates from diagonal two-cycles $q_{13}, q_{14}$, cf. Fig. 1d. If it so happens that more than one of the operations $q_{j}(j=3, \ldots, 14)$ are applicable to the old configuration we generally select the one with lowest index $j$. The reader may convince himself that we have ordered these operations such that the firstcoming successful one does not produce any dimers. As a rule there is at most one $q_{j}$ which does not produce dimers. The only exception to the latter statement results from the situation, where the plaquette is empty (all four dogs at home). Then both $q_{3}$ and $q_{4}$ lead to an allowed new permutation, since they can make a clockwise or counterclockwise permutation, respectively, cf. Fig. 3a. Now the reason for excluding a further production of dimers by one of the operators $q_{3} \ldots q_{14}$ is the following. Whenever possible we prefer a simple alternative (retain $c_{0}$ or take $c_{n}$ ) in the generation of configurations (but no more competing new configurations $\left.c_{n}^{\prime}, c_{n}^{\prime \prime}, \ldots\right)$. Namely, for the case of a simple alternative the probability formula (3.15) implies detailed balance, i.e. for an ensemble of configurations with Gibbs distribution $\propto e^{-\beta \ell}$ the flux from any configuration to another one is equal to the reverse one. In our algorithm there are always only simple alternatives except for the operations $q_{3}$ and $q_{4}$ as was mentioned above. If they are to be applied to an empty plaquette we first toss a coin between $q_{3}$ and $q_{4}$, i.e. with weight $1 / 2$ we choose randomly the direction of the cyclic permutation, and only afterwards apply the probability formula (3.15) to decide whether we actually accept the new configuration. For the reverse process of annihilating a $1 \times 1$ square loop we have to reduce the probability (3.15) by a factor of $1 / 2$ in order to preserve detailed balance.

The reader may convince himself that "ergodicity" of our upgrading procedure (apparently) is fulfilled: any allowed loop configuration ${ }^{1}$ on a $d$-dimensional hypercubic lattice can in principle be constructed (and again decomposed) after sufficiently many iterations of local changes as depicted in Figs. 2 and 3. Hence we expect that the so obtained Gibbs distribution is the unique equilibrium distribution for the upgrading procedure. Furthermore, starting from an arbitrary initial configuration we expect to reach this equilibrium after an appropriately long "warming up" period. From the sequence of configurations generated by iterations of the procedure just explained we then select a subset of configurations which are separated by so many single steps that their correlations (in the sense that the configurations may still "look very similar") seem negligible. We have performed a systematic investigation of such correlations only for some particular cases. Therefrom we got the impression that after two or three complete sweeps through the entire lattice we may neglect the correlations.

The thermal average $\bar{X}$ of a variable $X$, cf. Eq. (3.8), is approximated by

$$
\bar{X} \approx \frac{1}{n} \sum_{i=1}^{n} X\left(c_{i}\right),
$$

1 We were unable to find a counterexample. Compare, however, also the remarks after Eq. (3.23) 
where the sum now extends over a sufficiently large sample of equilibrium configurations $c_{i}$ generated according to the above prescription. Practically, our measurements at different values of the parameters $m$, respectively $k$, are performed in so-called thermal cycles. Starting with the empty configuration at a low "temperature" (i.e. small hopping parameter $k$, large mass $m$ ) we increment $k$ by a series of small steps up to a maximal value and then similarly return to the minimal value. The idea behind this is that equilibrium configurations do not vary too much for only gradually different temperatures. Of course, an appropriate warming up (or cooling down) period has to be included before an average (3.22) is taken at the new value.

Let us include at this stage some remarks on boundary conditions. In most of our calculations we take an "average" of periodic and antiperiodic boundary conditions, e.g.

$$
\operatorname{det}=\frac{1}{2}\left(\operatorname{det}_{\text {periodic }}+\operatorname{det}_{\text {antiperiodic }}\right) \text {, }
$$

the reason being that in this combination the contributions of loops which are topologically nontrivial (e.g. wrapping around the entire lattice) cancel out. Such nontrivial loops cannot be generated locally. We have also done some computations including such loops but in the present applications of our method they seem to be negligible.

At the end of this section we would like to explain how our heat bath method can be adapted for the calculation of fermionic Green's functions $\left\langle\psi_{x_{0}} \ldots \psi_{x_{n}}^{+}\right\rangle$. For simplicity we consider here only the two-point function

$$
\left\langle\psi_{v} \psi_{u}^{+}\right\rangle=(\gamma \partial+m)_{v u}^{-1},
$$

the generalizations being obvious. We consider a matrix $(\gamma \partial+m)^{(u)}$ which is equal to $\gamma \partial+m$, except that the row $u$ has been replaced by a row of 1's, i.e.

$$
(\gamma \partial+m)_{x y}=\left(1-\delta_{x, u}\right)(\gamma \partial+m)_{x y}+\delta_{x, v},
$$

which is a generalization of (2.7). The determinant of the modified matrix is easily calculated by expansion with respect to the row $u$,

$$
\operatorname{det}(\gamma \partial+m)^{(u)}=\operatorname{det}(\gamma \partial+m) \sum_{v}(\gamma \partial+m)_{v u}^{-1} .
$$

The auxiliary statistical mechanics system corresponding to $(\gamma \partial+m)^{(u)}$ again is a gas of self-avoiding closed oriented loops. In general, however, the configurations contain a particular closed loop which consists of a special line from the point $u$ to any point $v$ (which need not be a nearest neighbor of $u$ ), and then is closed by a path of ordinary links from $v$ to $u$. Since we have chosen all entries in the modified row to be equal to 1 , the energy of the special line is independent of its geometrical length. Therefore, we may disregard it altogether and say the configurations contain in addition to closed loops also an open line starting anywhere in the lattice and ending at the prescribed point $u$. In terms of our picture this means that the dog with home site $u$ has been replaced by a "cat" which is not confined to its neighborhood by a chain, hence it may displace a dog from any site. The cat, however, can also easily be confined to a certain range $I$ of points. In our computations we considered three different cases of intervals: the whole lattice 


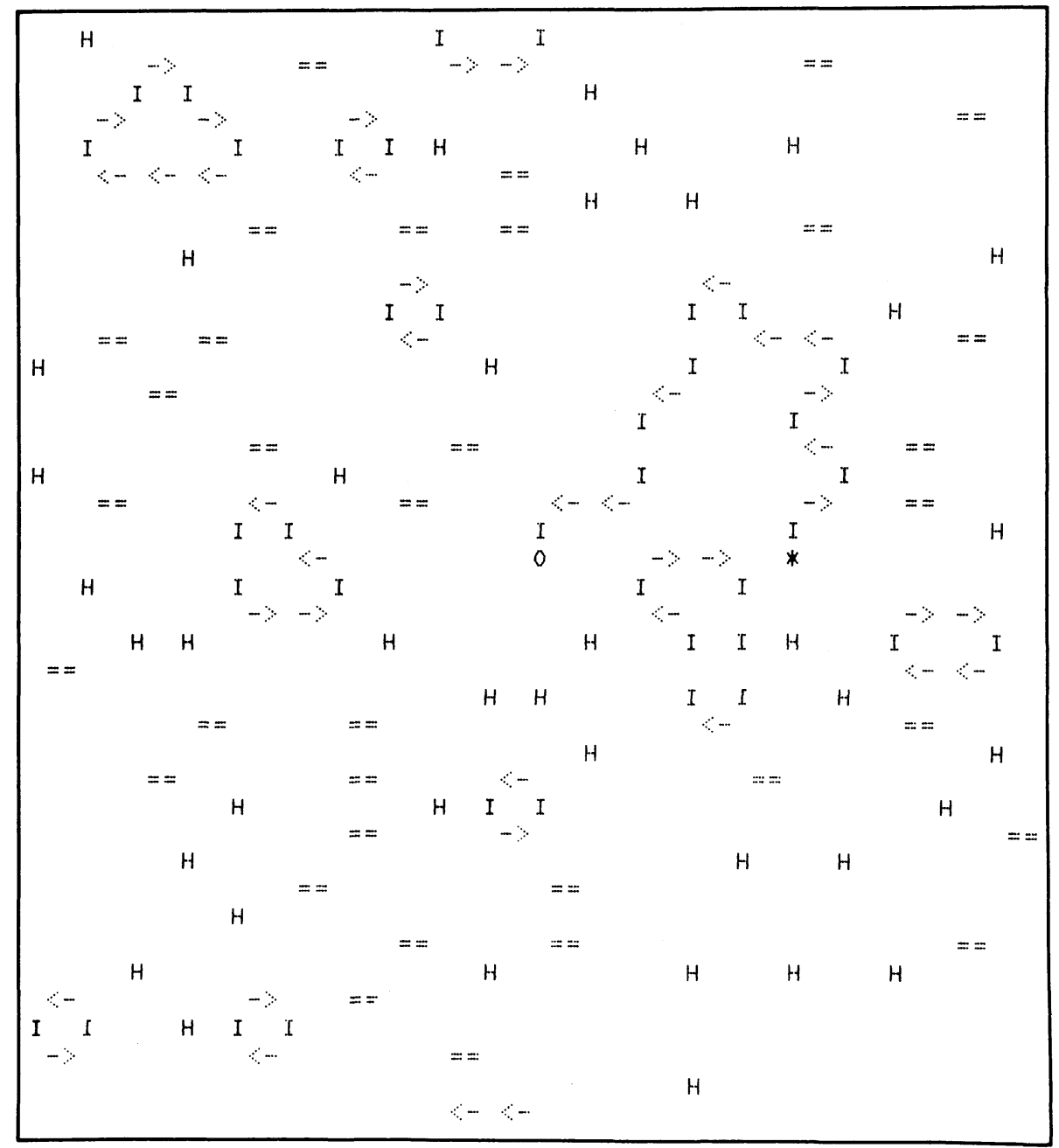

Fig. 4. A typical loop configuration including an open line starting at $*$ and ending at 0 . Dimers in the vertical or horizontal direction are represented by $H$ and $==$, respectively. The configuration was obtained in a Monte Carlo run for a free fermion with mass $m=1 /(2 k)$ for $k=0.6$ on a $20 \times 20$ lattice

$I=(0, N-1)$, a ray parallel to an axis, e.g. $I=(0, L-1)$, or a half-ray e.g. $I=(u+1, L-1)$. To this end all entries in row $u$ of $(\gamma \partial+m)_{x y}^{(u, I)}$ which correspond to forbidden sites, $y \notin I$, have to be set equal to zero, and Eqs. (3.25) and (3.26) have to be modified appropriately. In the process of generating new configurations we obviously can distinguish from each other the configurations which contribute to different terms on the right-hand side of Eq. (3.26), cf. Fig. 4. In terms of the characteristic function

$$
h(v, u)= \begin{cases}1 & \text { if the open line starts at } v, \\ 0 & \text { otherwise }\end{cases}
$$


we obtain

$$
\left\langle\psi_{v} \psi_{u}^{+}\right\rangle \propto \overline{h(v, u) \cdot \operatorname{sgn}(\gamma \partial+m)^{(u, I)}} \text { for } v \in I .
$$

The normalization factor may be determined from $\operatorname{det}(\gamma \partial+m)$, cf. Eq. (3.14),

$$
\begin{aligned}
m \sum_{x}\left\langle\psi_{x} \psi_{x}^{+}\right\rangle & =m \operatorname{tr}(\gamma \partial+m)^{-1} \\
& =m \frac{\partial}{\partial m} \ln \operatorname{det}(\gamma+m) \\
& =N-\langle\langle\ell\rangle .
\end{aligned}
$$

The upgrading procedure is modified as follows. Before every new sweep through the lattice we give the starting point of the open line (i.e. the cat) twice the chance to hop one step in any direction within the allowed range. We take the new configuration with probability $w_{n} /\left(w_{0}+w_{n}\right)$. The ratio of the Boltzmann weights is given by $w_{n} / w_{0}=k^{ \pm 1}$ if the length of the open line increases or decreases, respectively. Only afterwards we sweep through the entire lattice with our local rearrangement operators but then we keep the starting point of the open line fixed.

\section{Application of the Method}

In this section we apply our method to the Scalapino-Sugar model [3]. This model is exactly soluble but nontrivial for a Monte Carlo simulation. Since all interesting quantities are exactly known, we thus obtain a good test of the efficiency of our method. The interaction of a (static) scalar real Bose field $\phi$ and a massive Fermi field $\psi$ is governed by the Lagrangian

$$
\mathscr{L}_{F, B}=\psi^{+}\left(\gamma \partial+m+g \phi^{2}\right) \psi+\phi^{2} .
$$

By integrating out the Bose degrees of freedom one realizes that this interacting field theoretic model is equivalent to a free fermion model with effective mass

$$
m_{\mathrm{eff}}=m+g / 2 \text {. }
$$

Indeed, one obtains

$$
\begin{aligned}
Z_{F B}(m, g) & =\int d \psi^{+} d \psi d \phi e^{-\int d x \mathscr{L}_{F B}} \\
& =Z_{F B}\left(m_{\mathrm{eff}}, 0\right)=\pi^{N / 2} Z_{F}\left(m_{\mathrm{eff}}\right),
\end{aligned}
$$

with $Z_{F}$ given by Eq. (3.6). The vacuum expectation value of $\phi^{2}$ can be expressed in terms of $\psi \psi^{+}$:

$$
\left\langle\phi_{x}^{2}\right\rangle=\frac{1}{2}\left(1+g\left\langle\psi_{x} \psi_{x}^{+}\right\rangle\right)
$$

The fermion two-point function (on a $d$-dimensional hypercubic lattice, $L^{d}=N$ ) can be evaluated by Fourier sums, e.g.

$$
\begin{aligned}
\left\langle\psi_{x} \psi_{y}^{+}\right\rangle & =\left(\gamma \partial+m_{\mathrm{eff}}\right)_{x y}^{-1} \\
& =\frac{1}{N} \sum_{k_{\mu}=0}^{L-1} e^{\frac{2 \pi i}{L} \mathbf{k} \cdot(\mathbf{x}-\mathbf{y})}\left[m_{\mathrm{eff}}+i \gamma_{\mu} \sin \frac{2 \pi}{L} k_{\mu}\right]^{-1}
\end{aligned}
$$


for periodic boundary conditions (for the antiperiodic case $k_{\mu}$ in the propagator has to be replaced by $k_{\mu}+\frac{1}{2}$ ). In $d=1$ dimension all quantities can be expressed in closed form in terms of Gegenbauer polynomials, e.g. for $L \geqq 4$ and $L$ even one obtains

$$
\begin{aligned}
\operatorname{det}(\partial+m) & =2^{1-L} \cosh L \varphi, \\
\left\langle\psi \psi^{+}\right\rangle & =\tanh L \varphi / \cosh \varphi, \\
\langle\ell\rangle & =L(1-\tanh \varphi \cdot \tanh L \varphi),
\end{aligned}
$$

where $\sinh \varphi=m_{\text {eff }}$.

Let us now address ourselves to the application of the heat bath method to this model. For the interacting case the formula (3.6) has to be replaced by [cf. Eqs. (2.6) and (2.14)]

$$
\begin{aligned}
Z_{F B} & =\int d \phi \operatorname{det}\left(\gamma \partial+m+g \phi^{2}\right) e^{-\sum_{x} \phi_{x}^{2}} \\
& =2^{-N} \int d \phi \sum_{c \in \mathscr{C}} \operatorname{sgn}\left(c, \gamma \partial+m+g \phi^{2}\right) \prod_{p(x)=0}\left(2 m+2 g \phi_{x}^{2}\right) e^{-\sum_{x} \phi_{x}^{2}},
\end{aligned}
$$

and the partition function $Z$ for the auxiliary problem is obtained by omitting the factor $\operatorname{sgn}(\cdot, \cdot)$ in the last equation. A fermion configuration $c \in \mathscr{C}$ again consists of self-avoiding closed loops made up of simple links (i.e. the chains of the displaced dogs as was explained in Sect. 3). The fermionic Boltzmann weight at a point $x$ is given by

$$
w_{F}(x)=\left\{\begin{array}{lll}
2 m+2 g \phi^{2}(x) & \text { for } & p(x)=0, \\
1 & \text { for } & p(x) \neq 0,
\end{array}\right.
$$

i.e. we obtain a factor $\left(2 m+2 g \phi^{2}(x)\right)$ for all those dogs which are at home, $\pi(x)=x$, cf. Eq. (3.18). On the other hand, the local weight to be used in the upgrading of the bosons is

$$
w_{B}(x)=e^{-\phi^{2}(x)} w_{F}(x)
$$

The local upgrading procedure for the fermions is completely analogous to the free case explained in Sect. 3. The probability for accepting a new configuration is still given by Eq. (3.15) with, however, the relative weights $w_{F}^{n}$ and $w_{F}^{0}$ given by $\prod_{x} w_{F}(x)$, where the product is only over the points which are affected by the tentative local change. After one Monte Carlo step for the fermions all $d(d-1) / 2$ plaquettes emanating from the lattice point $x$ we upgrade the Bose field $\phi(x)$ by a modified Metropolis algorithm [40]. We propose a new field,

$$
\phi_{n}(x)=\phi_{0}(x)+r
$$

with a random number $r$ uniformly distributed in the interval $(-1,1)$. The new field is accepted with probability

$$
w=\max \left(1, w_{B}^{n}(x) / w_{B}^{0}(x)\right) .
$$




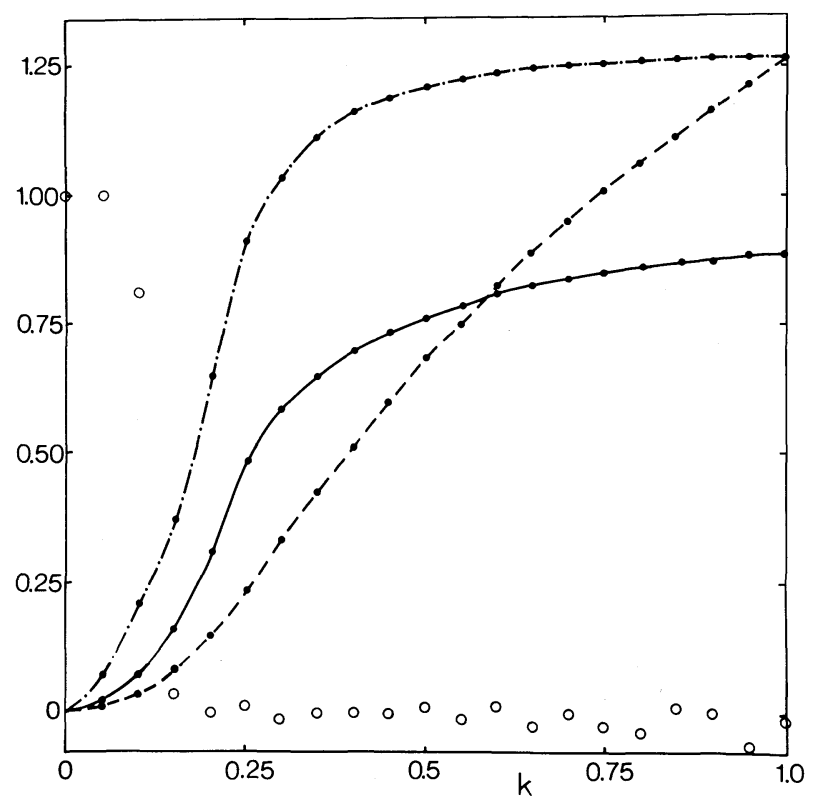

g. 5. Monte Carlo results for the thermal average values per lattice site of the length $\bar{\ell} / N(-)$, $(\log Z) / N(-\cdot-\cdot)$, and the entropy $S / N(--)$ versus $k=1 /(2 m)=e^{-1 / T}$ for the auxiliary statistical loop gas system corresponding to a free fermion on a $8^{4}$ lattice. In addition $\overline{\operatorname{sgn}}(\mathrm{O})$ is plotted

We repeat this bose upgrading five times before passing to the next point.

Let us finally report our results. We considered hypercubic lattices in 1, 2, 3, and 4 dimensions with linear sizes $L=100-1000,4-40,4-16$, and 4-10, respectively. By means of Monte Carlo simulations in thermal cycles $0 \leqq k \leqq k_{\max }$ for various numbers of intermediate steps and values for $k_{\max }$, we generated samples of equilibrium ensembles of fermion-boson configurations $(c, \phi)$ in two series of runs:

(a)

$$
(c, \phi) \in \mathscr{C}\left(\gamma \partial+m+g \phi^{2}\right) \otimes \mathbb{R}^{N},
$$

i.e. $c$ is a configuration of closed self-avoiding oriented loops.

$$
(c, \phi) \in \mathscr{C}\left(\gamma \partial+m+g \phi^{2}\right)^{(u, I)} \otimes \mathbb{R}^{N},
$$

i.e. $c$ contains in addition to case (a) one self-avoiding open line starting at some $v \in I$ and ending at $u$, which also avoids the loops.

For the case (a) we "measured" using Eq. (3.22) the auxiliary quantities $\bar{\ell}$, $\overline{\operatorname{sgn}(A)}, \overline{\ell \operatorname{sgn}(A)}$, and $\overline{\phi^{2} \operatorname{sgn}(A)}$ for the matrix $A=\gamma \partial+m+g \phi^{2}$. From the average energy $\bar{\ell}$ at temperature $T=-1 / \ln k$ (for a sufficiently large number of $k$-values with $0 \leqq k \leqq k_{\max }$ ) we can calculate other thermodynamic quantities for our statistical system. For the free fermion case, $g=0$, we then obtain by numerical integration of Eq. (3.12) the partition function $Z$ and therefrom the entropy $S=\ln Z+\bar{\ell} / T$, see Fig. 5. For infinite temperature some results for the entropy per lattice site are listed in Table 1 for various dimensions and lattice sizes. Note that finite size effects seem negligible and a good fit is $S / N \approx(d+1) / 4$. Therefore, the 
Table 1. Monte Carlo results for the entropy per lattice site $S / N$ at infinite temperature (or mass $=1 / 2$ ) for loop gas systems corresponding to free fermion field theories on various lattices $L^{d}$

\begin{tabular}{|c|c|c|c|c|c|c|c|c|c|c|c|c|}
\hline \multirow{2}{*}{$\frac{d}{L}$} & \multicolumn{2}{|l|}{1} & \multicolumn{4}{|l|}{2} & \multicolumn{3}{|l|}{3} & \multicolumn{3}{|l|}{4} \\
\hline & 100 & 1000 & 4 & 10 & 20 & 40 & 4 & 8 & 16 & 4 & 6 & 8 \\
\hline$S / N$ & 0.479 & 0.478 & 0.764 & 0.763 & 0.762 & 0.764 & 1.02 & 1.03 & 1.03 & 1.25 & 1.26 & 1.26 \\
\hline
\end{tabular}

total number of loop configurations for dimensions $d \leqq 4$ is approximately given by $\exp (N \cdot(d+1) / 4)$.

The "physical" quantities $\left\langle\psi \psi^{+}\right\rangle$and $\left\langle\phi^{2}\right\rangle$ (see Fig. 6) are obtained by Eqs. (3.10), (3.14), and (3.29), and the fermion determinant $Z_{F}=\operatorname{det}(\gamma \partial+m)$ for the free case $g=0$ by Eq. (3.13) or by Eqs. (3.7) and (3.9).

We have two types of checks of our Monte Carlo results,

i) consistency checks e.g.

$$
\begin{gathered}
\left\langle\phi^{2}\right\rangle=\left(1+g\left\langle\psi \psi^{+}\right\rangle\right) / 2, \quad \text { cf. (4.4), } \\
Z_{F}=m^{N} Z \overline{\operatorname{sgn}}, \quad \text { cf. (3.9), }
\end{gathered}
$$

ii) comparison with numbers obtained by numerical Fourier summation, cf. Eq. (4.5).

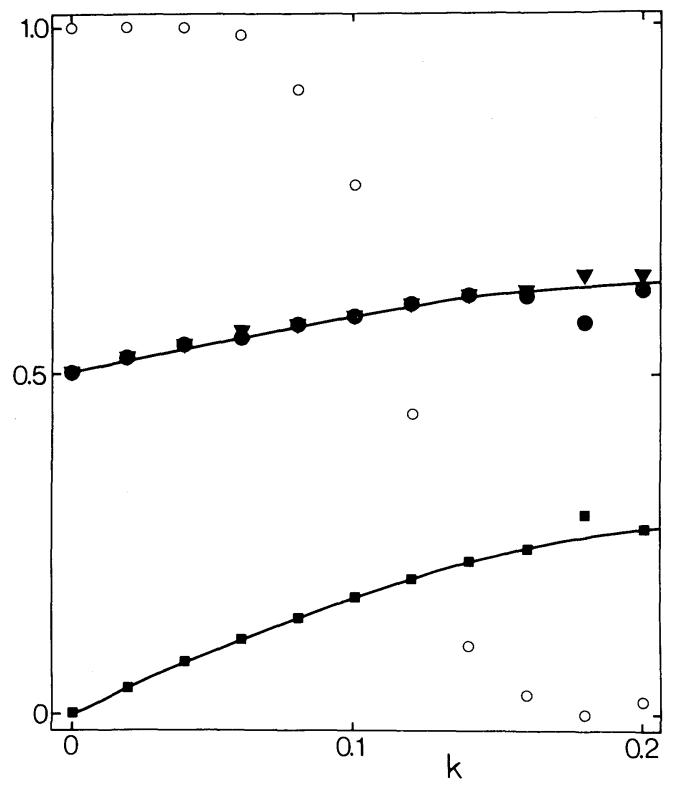

Fig. 6. Monte Carlo results for $\left\langle\psi \psi^{+}\right\rangle(\mathbf{\bullet})$ and $\left\langle\phi^{2}\right\rangle(\bullet)$ versus the hopping parameter $k=1 /(2 m)$ for the Scalapino-Sugar model with coupling $g=1$ on a $10^{4}$ lattice. The curves are drawn through the values obtained by Fourier summation. For a check of Eq. (4.4) the quantity $\left(1+g\left\langle\psi \psi^{+}\right\rangle\right) / 2(\nabla)$ is included, too. The auxiliary quantity $\overline{\operatorname{sgn}}(0)$ is also plotted 
Table 2. Monte Carlo results for the fermion determinant $Z_{F}$ in terms of $A=\ln \left(Z_{F}^{\mathrm{MC}} / m^{N}\right) / N$, as a check $B=\ln \left(Z^{\mathrm{MC}}(\overline{\mathrm{sgn}})^{\mathrm{MC}}\right) / N$, and the "physical" quantity $\left\langle\psi \psi^{+}\right\rangle^{\mathrm{MC}}$ in comparison with Fourier results $\left\langle\psi \psi^{+}\right\rangle^{\text {Fou }}$ for free fermions with mass $m=1 /(2 k)$ on various lattices $L^{d}$

\begin{tabular}{lllllll}
\hline$d$ & $L$ & $k$ & $A$ & $B$ & $\left\langle\psi \psi^{+}\right\rangle^{\mathrm{MC}}$ & $\left\langle\psi \psi^{+}\right\rangle^{\text {Fou }}$ \\
\hline 1 & 100 & 0.5 & 0.188 & 0.188 & 0.704 & 0.707 \\
1 & 100 & 5 & 1.71 & 1.71 & 1.05 & 1.00 \\
2 & 20 & 0.2 & 0.0727 & 0.0727 & 0.346 & 0.347 \\
2 & 20 & 1 & 0.786 & 0.753 & 0.50 & 0.51 \\
3 & 16 & 0.1 & 0.0288 & 0.0288 & 0.191 & 0.189 \\
3 & 16 & 0.25 & 0.158 & 0.157 & 0.359 & 0.368 \\
4 & 8 & 0.1 & 0.0381 & 0.0381 & 0.187 & 0.185 \\
4 & 8 & 0.15 & 0.0808 & 0.0818 & 0.256 & 0.255 \\
\hline
\end{tabular}

Table 3. Monte Carlo results and checks for the Scalapino-Sugar model with mass $m=1 /(2 k)$ and coupling $g=1$ on various lattices $L^{d}$

\begin{tabular}{lllllll}
\hline$d$ & $L$ & $k$ & $\left\langle\psi \psi^{+}\right\rangle^{\mathrm{MC}}$ & $\left\langle\psi \psi^{+}\right\rangle^{\mathrm{Fou}}$ & $\left\langle\phi^{2}\right\rangle^{\mathrm{MC}}$ & $\left(1+g\left\langle\psi \psi^{+}\right\rangle\right) / 2$ \\
\hline 1 & 1000 & 0.5 & 0.5548 & 0.5547 & 0.7769 & 0.7774 \\
1 & 1000 & 5 & 0.8581 & 0.8575 & 0.9284 & 0.9290 \\
2 & 20 & 0.2 & 0.3010 & 0.3008 & 0.6486 & 0.6505 \\
2 & 20 & 1.0 & 0.5130 & 0.5136 & 0.7554 & 0.7565 \\
3 & 16 & 0.1 & 0.1737 & 0.1733 & 0.5868 & 0.5866 \\
3 & 16 & 0.25 & 0.3227 & 0.3246 & 0.6614 & 0.6623 \\
4 & 10 & 0.1 & 0.1698 & 0.1706 & 0.5842 & 0.5849 \\
4 & 10 & 0.2 & 0.2749 & 0.2739 & 0.623 & 0.637 \\
\hline
\end{tabular}

In $d=1$ dimensions we have also the exact formulae (4.6). In Tables 2 and 3 we list some comparable quantities for various dimensions and lattice sizes for $g=0$ and $g=1$, respectively. The suffixes $\mathrm{MC}$ and Fou are employed to distinguish Monte Carlo from Fourier results. As a rule we find agreement within a few percent

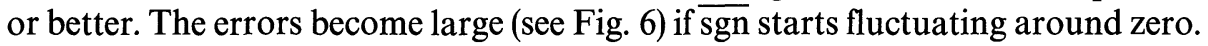
This phenomenon determines the critical values of $k$ which restricts the applicability of our Monte Carlo method to small $k$, as was already mentioned at the beginning of Sect. 3 .

For the case (b) we "measured" the two point function $\left\langle\psi_{v} \psi_{u}^{+}\right\rangle$using Eq. (3.28). The results are in good agreement with the Fourier results, cf. Figs. 7 and 8 . The exponential decay can be fitted by the ansatz

$$
\left\langle\psi_{v} \psi_{u}^{+}\right\rangle \sim e^{-\mu|\mathbf{v}-\mathbf{u}|} .
$$

In Table 4 we have collected the corresponding mass parameters. Again the agreement is satisfactory. Note that the parameter $\mu$ differs from the effective mass of the model if the latter is not small compared to the cut off mass $1 /$ (lattice spacing) which has been set equal to 1 . 


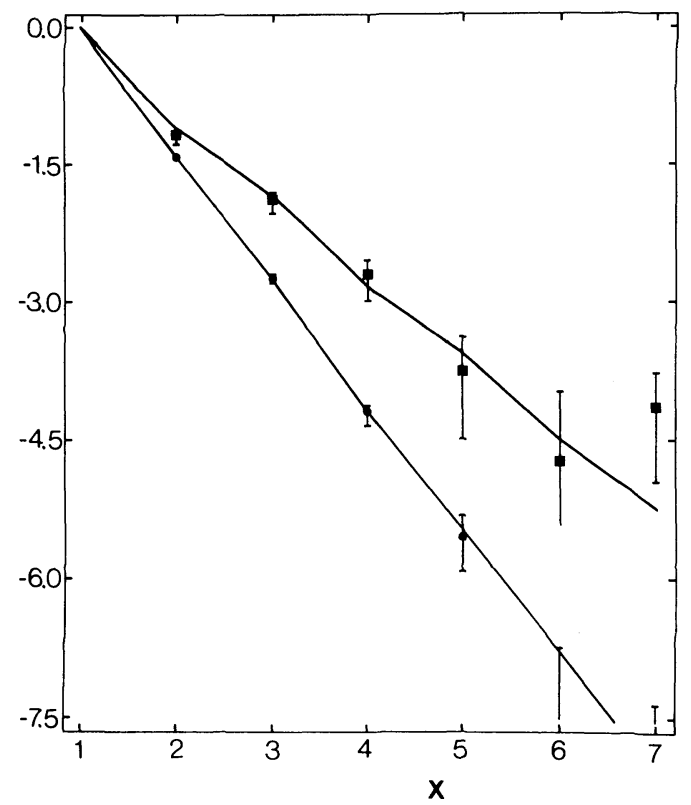

Fig. 7. Monte Carlo results for $\log \left(\left\langle\psi(x) \psi^{+}(0)\right\rangle /\left\langle\psi(1) \psi^{+}(0)\right\rangle\right)$ versus the distance $x$ for a free fermion with mass $m=1 /(2 k)$ and $k=0.3(\bullet)$, and $k=0.6(\boldsymbol{\bullet})$ on a $20 \times 20$ lattice. The solid lines connect the values obtained by Fourier summation. A typical configuration for $k=0.6$ is shown in Fig. 4

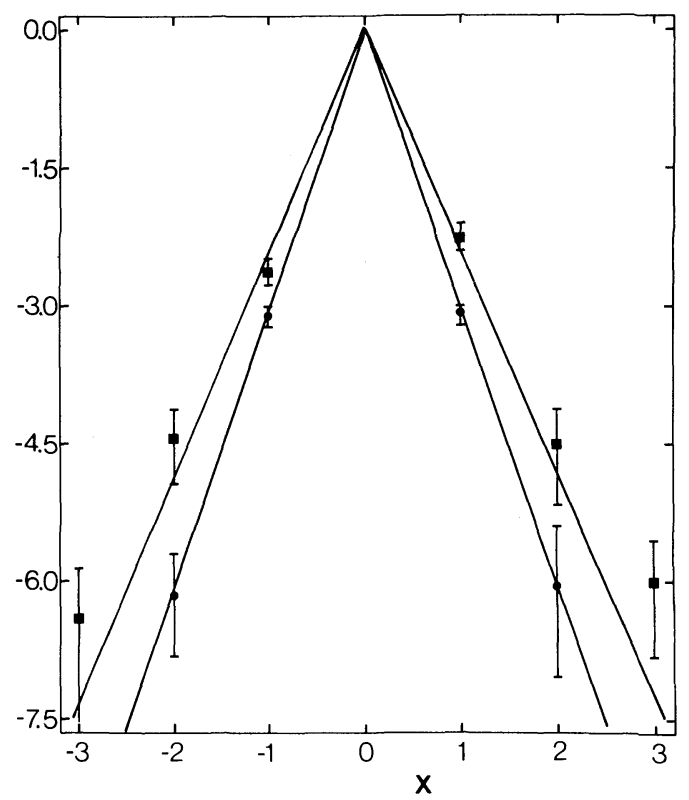

Fig. 8. Monte Carlo results for $\log \left(\left\langle\psi(x) \psi^{+}(0)\right\rangle /\left\langle\psi(0) \psi^{+}(0)\right\rangle\right)$ versus the distance $x$ for the Scalapino-Sugar model with $k=1 /(2 m)=0.05(\bullet)$ and $0.1(\boldsymbol{\bullet})$ and coupling $g=1$ on a $10^{4}$ lattice. The solid lines connect the values obtained by Fourier summation 
Table 4. Monte Carlo results for the mass parameter $\mu$ obtained from the fit Eq. (4.20) in comparison with Fourier results for the Scalapino-Sugar model with mass $m=1 /(2 k)$ and coupling $g$ on various lattices $L^{d}$

\begin{tabular}{lllllll}
\hline$d$ & $L$ & $g$ & $k$ & $m_{\text {eff }}$ & $\mu^{\text {MC }}$ & $\mu^{\text {Fou }}$ \\
\cline { 2 - 6 } 1 & 100 & 0 & 1 & 0.5 & 0.487 & 0.481 \\
1 & 100 & 0 & 2 & 0.25 & 0.253 & 0.247 \\
1 & 100 & 1 & 1 & 1.0 & 0.878 & 0.881 \\
1 & 100 & 1 & 2 & 0.75 & 0.693 & 0.693 \\
2 & 20 & 0 & 0.3 & 1.67 & 1.36 & 1.35 \\
2 & 20 & 0 & 0.6 & 0.83 & 1.0 & 0.9 \\
2 & 20 & 1 & 0.3 & 2.17 & 1.58 & 1.56 \\
2 & 20 & 1 & 0.6 & 1.33 & 1.18 & 1.18 \\
3 & 16 & 0 & 0.1 & 5 & 2.38 & 2.33 \\
3 & 16 & 0 & 0.2 & 2.5 & 1.26 & 1.70 \\
3 & 16 & 1 & 0.1 & 5.5 & 2.33 & 2.41 \\
3 & 16 & 1 & 0.2 & 3 & 1.75 & 1.85 \\
4 & 10 & 0 & 0.05 & 10 & 2.89 & 3.00 \\
4 & 10 & 0 & 0.1 & 5 & 2.30 & 2.35 \\
4 & 10 & 1 & 0.05 & 10.5 & 3.10 & 3.05 \\
4 & 10 & 1 & 0.1 & 5.5 & 2.42 & 2.41 \\
\hline
\end{tabular}

\section{Conclusions}

In this paper we have introduced a new method for numerical investigations of quantum field theories with fermions. The essential point is the construction of a "heat bath for fermion determinants" on a lattice. For simplicity we restricted our discussion to the case of Susskind fermions and hypercubic lattices. The key observation relies on the fact that every term contributing to the expansion of a determinant may be graphically represented by a configuration of self-avoiding oriented loops. A direct application of importance sampling by a Monte Carlo procedure is inhibited by the different signs with which these configurations contribute to the determinant. We therefore introduce an auxiliary thermodynamical system of a gas of self-avoiding oriented loops with Boltzmann weights proportional to the modulus of the above contributions. The sign is then subsumed in the observables. Therefore, the quantum field expectation values turn out to be ratios of thermodynamic average values.

We have devised a local upgrading procedure for generating equilibrium ensembles of loop configurations. The boson fields can be treated as usual in Monte Carlo simulations. This allows us to evaluate bosonic physical quantities in quantum field theories involving fermions. By introducing one open line besides the loops we calculate fermionic Green's functions.

In most previous proposals the fermions are either not considered as dynamical variables at all or they require a nonlocal upgrading. In contrast we include all fermionic vacuum fluctuations, treat fermions and bosons on the same footing and work with a local upgrading procedure. The computer times for the fermionic and the bosonic degrees of freedom are comparable. In the application to the Scalapino-Sugar model we found good agreement with known results. 
We plan to extend our calculations to more interesting field theoretic models involving fermions, including gauge theories. Instead of Susskind fermions we then shall use Wilson fermions in order to suppress the extra light fermions which are responsible for large statistical errors in some of our present computations.

In Sect. 2 we also mentioned how by a modification of our techniques, one obtains a description and an upgrading procedure for the statistical ensemble of a polymer gas. In fact, this method was used in [42] to obtain numerical information on its critical behaviour, see also [9].

By extending the ideas developed there, a numerical discussion of the theory of self-avoiding random surfaces is given in [43], which differs from the upgrading procedure used in [44] (see also [45]). We emphasize that although we designed our method with local lattice theories in mind, it could also have other applications like calculating Slater determinants in nuclear and condensed matter physics. Finally, $[46,47]$ give a discussion of "euclidean" lattice gravity using Regge calculus. There it is pointed out that already this lattice version contains a Faddeev-Popov determinant which necessitates the introduction of fermionic and bosonic ghost fields to restore locality. In principle our method should also be able to treat these fermionic fields numerically.

Acknowledgements. We thank B. Schroer for discussions. One of us (M.K.) thanks C. N. Yang for financial support for a visit to and one of us (R.S.) for kind hospitality at the Institute of Theoretical Physics (SUNY), Stony Brook. This work was supported by the Deutsche Forschungsgemeinschaft under contracts Schr 160/7-1 and Schr 4/9-2 and by NSF Grant Phy 8109110 A 01. The numerical calculations were performed on the VAX 11/780 computers of Fachbereich Physik der Freien Universität Berlin and Gesellschaft für wissenschaftliche Datenverarbeitung $\mathrm{mbH}$, Göttingen.

\section{References}

1. Weingarten, D.H., Petcher, D.N.: Monte Carlo integration for lattice gauge theories with fermions. Phys. Lett. 99 B, 333 (1981)

2. Fucito, F., Marinari, E., Parisi, G., Rebbi, C.: A proposal for Monte Carlo simulations of fermionic systems. Nucl. Phys. B 180, 369 (1981)

3. Scalapino, D.J., Sugar, R.L.: Method for performing Monte Carlo calculations for systems with fermions. Phys. Rev. Lett. 46, 519 (1981)

4. Hamber, H.: Monte Carlo simulations of systems with fermions. Phys. Rev. D 24, 951 (1981)

5. Marinari, E., Parisi, G., Rebbi, C.: Monte Carlo simulation of the massive Schwinger model. Nucl. Phys. B 190, 734 (1981)

6. Duncan, F., Furman, A.: Monte Carlo calculations with fermions: the Schwinger model. Nucl. Phys. B 190, 767 (1981)

7. Blankenbecler, R., Scalapino, D.J., Sugar, R.L.: Monte Carlo calculations of coupled bosonfermion systems. I. Phys. Rev. D 24, 2278 (1981)

8. Hirsch, J., Scalapino, D.J., Sugar, R.L., Blankenbecler, R.: Efficient Monte Carlo procedure for systems with fermions. Phys. Rev. Lett. 47, 1628 (1981)

9. Berg, B., Foerster, D.: Random paths and random surfaces on a digital computer. Phys. Lett. $106 \mathrm{~B}, 323$ (1981)

10. Hamber, H., Parisi, G.: Numerical estimates of hadronic masses on a pure $S U(3)$ gauge theory. Phys. Rev. Lett. 47, 1792 (1981)

Hamber, H., Marinari, E., Parisi, G., Rebbi, C.: Spectroscopy in a latter gauge theory. Phys. Lett. 108 B, 314 (1982) 
11. Weingarten, D.H.: Monte Carlo evaluation of hadron masses in lattice gauge theories with fermions. Phys. Lett. 109 B, 57 (1982)

12. Hasenfratz, A., Hasenfratz, P., Kunszt, Z., Lang, C.B.: Hopping parameter expansion for the meson spectrum in $S U(3)$ lattice QCD. Phys. Lett. 110 B, 289 (1982)

13. Kuti, J.: Stochastic method for the numerical study of lattice fermions. Phys. Rev. Lett. 49, 183 (1982)

14. Anthony, S.J., Llewellyn Smith, C.H., Wheater, J.F.: A new proposal for Monte Carlo simulation of fermions on a lattice. Phys. Lett. 116 B, 287 (1982)

15. Duffy, W., Guralnik, G., Weingarten, D.: Error estimate for the valence approximation to lattice QCD. Bloomington preprint (1983)

16. Joos, H., Montvay, I.: The screening of colour charge in numerical hopping parameter expansion. Nucl. Phys. B 225 [FS9], 565 (1983)

17. Bhanot, J.: Lattice gauge theory: the Monte Carlo approach. CERN preprint TH. 3507 (1983)

18. Creutz, M., Jacobs, L., Rebbi, C.: Monte Carlo computations in lattice gauge theories. Brookhaven preprint BNL 32438 (1983)

19. Rebbi, C.: Lattice gauge theories and Monte Carlo simulations. Singapore: World Scientific 1983

20. Symanzik, K.: Euclidean quantum field theory. I. Equations for a scalar model. J. Math. Phys. 7, $510(1966)$

21. Symanzik, K.: In, Proc. Intern. School of Physics "Enrico Fermi", Varenna Course XLV, Jost, R. (ed.). New York: Academic Press 1969

22. Kogut, J., Susskind, L.: Hamiltonian formulation of Wilson's lattice gauge theories. Phys. Rev. D 11, 395 (1975)

23. Berezin, F.A.: The method of second quantization. New York, San Francisco, London: Academic Press 1966

24. Fisher, M.E.: Statistical mechanics of dimers on a plane lattice. Phys. Rev. 124, 1664 (1961)

25. Temperley, H.N.V., Fisher, M.E.: Dimer problem in statistical mechanics - an exact result. Philos. Mag. 6, 1061 (1961)

26. Kasteleyn,P.W.: The statistics of dimers on a lattice. I. The number of dimer arrangements on a quadratic lattice. Physica 27, 1209 (1961)

27. Fisher, M.E., Stephenson, J.: Statistical mechanics of dimers on a plane lattice. II. Dimer correlations and monomers. Phys. Rev. 132, 1411 (1963)

28. Brydges, D., Fröhlich, J., Spencer, T.: The random walk representation of classical spin systems and correlation inequalities. Commun. Math. Phys. 83, 123 (1982)

29. Aizenman, M.: Geometric analysis of $\phi^{4}$ fields and Ising models. Parts I and II. Commun. Math. Phys. 86, 1 (1982)

30. Wilson, K.: Confinement of quarks. Phys. Rev. D 10, 2445 (1974)

Wilson, K.: Quarks on a lattice, or, the colored string model. Phys. Reports 23, 331 (1975) Wilson, K.: In: New phenomena in subnuclear physics, Zichichi, A. (ed.). New York: Plenum Press 1977

31. Kogut, J., Susskind, L.: Hamiltonian formulation of Wilson's lattice gauge theories. Phys. Rev. D 11, 395 (1975)

32. Banks, T., Raby, S., Susskind, L., Kogut, J., Jones, D.R.T., Scharbach, P., Sinclair, D.: Strongcoupling calculations of the hadron spectrum of quantum chromodynamics. Phys. Rev. D 15, 1111 (1977)

33. Susskind, L.: Lattice fermions. Phys. Rev. D 16, 3031 (1977)

34. Sharatchandra, H.S., Thun, H.J., Weisz, P.: Susskind fermions on a Euclidean lattice. Nucl. Phys. B 192, 205 (1981)

35. Kawamoto, N., Smit, J.: Effective lagrangian and dynamical symmetry breaking in strongly coupled lattice QCD. Nucl. Phys. B 192, 100 (1981)

36. Kluberg-Stern, H., Morel, A., Napoly, O., Petersson, B.: Flavours of Lagrangean Susskind fermions. Nucl. Phys. B 220 [FS8], 447 (1983)

37. Becher, P.: Dirac fermions on the lattice - a local approach without spectrum degeneracy. Phys. Lett. 104 B, 221 (1981) 
38. Becher, P., Joos, H.: The Dirac-Kähler equation and fermions on a lattice. Z. Phys. C 15, 343 (1982)

39. Mitra, P.: Geometry of non-degenerate Susskind fermions. Nucl. Phys. B 227, 349 (1983)

40. Metropolis, N., Rosenbluth, A.W., Rosenbluth, M.N., Teller, A.H., Teller, E.: Theory of the effects of exchange on the nuclear fine structure in the paramagnetic resonance spectra of liquids. J. Chem. Phys. 27, 1087 (1953)

41. Friedberg, R., Cameron, J.: Test of the Monte Carlo method: Fast simulation of a small Ising lattice. J. Chem. Phys. 52, 6049 (1970)

42. Karowski, M., Thun, H.J., Helfrich, W., Rys, F.: Numerical study of self-avoiding loops on $d$-dimensional hypercubic lattices. J. Phys. A 16, 4073 (1983)

43. Karowski, M., Thun, H.J.: Monte Carlo simulations for self-avoiding surfaces (in preparation)

44. Sterling, T., Greensite, J.: Entropy of self-avoiding surfaces on the lattice. Phys. Lett. 121 B, 345 (1983)

45. Schrader, R.: On the Euler characteristics of random surfaces. Stony Brook preprint ITP-SB-84-44 (1984)

46. Cheeger, J., Müller, W., Schrader, R.: In: Unified theories of elementary particles (Heisenberg Symposium 1981), Breitenlohner, P., Dürr, H.P. (eds.). Lecture Notes in Physics, Vol. 160. Berlin, Heidelberg, New York: Springer 1982

47. Cheeger, J., Müller, W., Schrader, R.: On the curvature of piecewise flat spaces. Commun. Math. Phys. 92, 405 (1984)

Communicated by G. Mack

Received February 7, 1984 
